\title{
Soft gluon resummation for squark and gluino pair-production at hadron colliders
}

\section{Wim Beenakker}

Theoretical High Energy Physics, Radboud University Nijmegen, P.O. Box 9010

NL-6500 GL Nijmegen, The Netherlands

E-mail: W.Beenakker@science.ru.nl

\section{Silja Brensing, Michael Krämer, Anna Kulesza*}

Institute for Theoretical Physics, RWTH Aachen University, D-52056 Aachen, Germany

E-mail: brensing@physik.rwth-aachen.de,

mkraemer@physik.rwth-aachen.de,

anna.kulesza@physik.rwth-aachen.de

\section{Eric Laenen}

ITFA, University of Amsterdam, Valckenierstraat 65, 1018 XE Amsterdam, The Netherlands

ITF, Utrecht University, Leuvenlaan 4, 3584 CE Utrecht, The Netherlands

Nikhef Theory Group, Science Park 105, 1098 XG Amsterdam, The Netherlands

E-mail: t45@nikhef.nl

\section{Leszek Motyka}

II Institute for Theoretical Physics, University of Hamburg, Luruper Chaussee 149, D-22761,

Germany

Institute of Physics, Jagellonian University, Reymonta 4, 30-059 Kraków, Poland

E-mail: motyka@mail.desy.de

\section{Irene Niessen}

Theoretical High Energy Physics, Radboud University Nijmegen, P.O. Box 9010

NL-6500 GL Nijmegen, The Netherlands

E-mail: i.niessen@science.ru.nl

We report on the study of soft gluon effects in the production of squarks and gluinos at hadron colliders. Close to production threshold, the emission of soft gluon results in the appearence of large logarithmic corrections in the theoretical expressions. In order to resum these corrections at next-to-leading-logarithmic accuracy appropriate one-loop anomalous dimensions have to be calculated. We present the calculation of the anomalous dimensions for all production channels of squarks and gluinos and provide numerical predictions for the Tevatron and the LHC.

RADCOR 2009 - 9th International Symposium on Radiative Corrections (Applications of Quantum Field Theory to Phenomenology)

October 25-30 2009

Ascona, Switzerland

\footnotetext{
${ }^{*}$ Speaker.
} 


\section{Introduction}

Extensions of the Standard Model (SM) involving supersymmetry (SUSY) are one of the best motivated models of new physics. The searches for signals of supersymmetry (SUSY) are currently carried out by the Tevatron experiments $[1,2]$ and in the coming years will be undertaken by the experiments at the Large Hadron Collider (LHC). Within the Minimal Supersymmetric Standard Model (MSSM) [3], the dominant production processes of sparticles at hadron colliders are those involving pairs of coloured particles, i.e. squarks and gluinos, in the final state. Depending on the outcome of the experimental searches, predictions for the total rates for these production processes are either used to draw the exclusion limits for the mass parameters or will help to determine the masses of the sparticles. It is therefore important to know the cross sections for hadroproduction of squarks and gluinos with high theoretical accuracy.

There are four pair-production processes of squarks and gluinos in hadronic collisions: $p \stackrel{(-)}{p} \rightarrow$ $\tilde{q} \tilde{\tilde{q}}, p \stackrel{(-)}{p} \rightarrow \tilde{q} \tilde{q}, p(\vec{p}) \rightarrow \tilde{g} \tilde{g}, p \stackrel{(-)}{p} \rightarrow \tilde{q} \tilde{g}$. The next-to-leading order (NLO) SUSY-QCD corrections [4, 5] to hadroproduction processes are generally positive at the central scale. Depending on the process and the masses of sparticles considered, they can be large. As pointed out in [5], an important part of the contributions to the hadronic cross sections comes from the energy region near the partonic production threshold, reached when the square of the partonic center-of-mass (c.o.m.) energy squared $\hat{s}$ approaches $4 m^{2}$, where $m$ is the average mass of the produced particles. In this region, two types of corrections dominate: the Coulomb corrections, due to exchange of gluons between slowly moving massive particles, and the soft-gluon corrections, due to emission of low-energetic gluons off the coloured initial and final states. The large size of the soft-gluon emission contributions can be traced back, for the perturbative $n$-th order correction, to the logarithmic terms of the form $\alpha_{\mathrm{s}}^{n} \log ^{k}\left(\beta^{2}\right)$ where $k=2 n, \ldots, 0$ with $\beta \equiv \sqrt{1-4 m^{2} / \hat{s}}$. The effects of the soft-gluon emission are taken into account to all orders in perturbation theory by performing resummation of the threshold logarithms.

Here we review calculations of the resummed total cross sections for the squark and gluino hadroproduction presented in $[6,7,8]$. The LO Coulomb corrections to $\tilde{q} \overline{\tilde{q}}$ and $\tilde{g} \tilde{g}$ production have been resummed in [7]. For the squark-antisquark production process the dominant contribution to the next-to-next-to-leading order (NNLO) correction coming from the resummed cross section at next-to-next-to-leading-logarithmic (NNLL) level has been studied in [9]. Recently, a general formalism allowing for simultaneous resummation of threshold and Coulomb corrections has been presented and applied to $\tilde{q} \overline{\tilde{q}}$ production [10].

\section{NLL threshold resummation}

The inclusive hadroproduction cross section $\sigma_{h_{1} h_{2} \rightarrow k l}$ for two massive SUSY particles $k$ and $l$, where $k, l$ can be a squark $(\tilde{q})$, antisquark $(\overline{\tilde{q}})$ or gluino $(\tilde{g})$, can be written in terms of its partonic version $\sigma_{i j \rightarrow k l}$ as

$\sigma_{h_{1} h_{2} \rightarrow k l}\left(\rho,\left\{\underline{m}^{2}\right\}\right)=\sum_{i, j} \int d x_{1} d x_{2} d \hat{\rho} \delta\left(\hat{\rho}-\frac{\rho}{x_{1} x_{2}}\right) f_{i / h_{1}}\left(x_{1}, \mu^{2}\right) f_{j / h_{2}}\left(x_{2}, \mu^{2}\right) \sigma_{i j \rightarrow k l}\left(\hat{\rho},\left\{\underline{m}^{2}\right\}, \mu^{2}\right)$, 
where $\left\{\underline{m}^{2}\right\}$ denotes all masses entering the calculations, $i, j$ are the initial parton flavours, $f_{i / h_{1}}$ and $f_{j / h_{2}}$ the parton distribution functions, and $\mu$ is the common factorization and renormalization scale. For the hadronic c.o.m. energy $S$, we define the threshold variable $\rho=\left(m_{k}+m_{l}\right)^{2} / S=$ $\mathrm{m}^{2} / S$. It measures the distance from threshold for inclusive production of two final-state particles with masses $m_{k}$ and $m_{l}$ in terms of the energy fraction. The partonic equivalent of this threshold variable is defined as $\hat{\rho}=\rho /\left(x_{1} x_{2}\right)$, where $x_{1,2}$ are the momentum fractions of the partons. The resummation of threshold logarithms is performed in the space of Mellin moments $N$ of the cross section (2.1), taken with respect to the variable $\rho$. Since the soft radiation carries colour charge, it can change the colour state of the underlying hard scattering. Consequently, the components in the resummed formula describing hard-scattering as well as the components corresponding to soft emission carry dependence on the colour configuration of all four particles participating in the reaction $i j \rightarrow k l$. This dependence can be vastly simplified if one performs the calculation in an orthogonal basis in the colour space for which the soft anomalous dimension matrices, governing the soft emission, are diagonal. In this case, up to next-to-leading-logarithmic (NLL) accuracy, the resummed partonic cross section reads $[11,12,13,14,15,16]$,

$$
\begin{aligned}
& \sigma_{i j \rightarrow k l}^{(\mathrm{res})}\left(N,\left\{\underline{m}^{2}\right\}, \mu^{2}\right)=\int_{0}^{1} d \hat{\rho} \hat{\rho}^{N-1} \sigma_{i j \rightarrow k l}\left(\hat{\rho},\left\{\underline{m}^{2}\right\}, \mu^{2}\right)= \\
& =\sum_{I} \tilde{\sigma}_{i j \rightarrow k l, I}^{(0)}\left(N, \alpha_{\mathrm{s}}\left(\mu^{2}\right)\right) \Delta_{i}\left(N+1, Q^{2}, \mu^{2}\right) \Delta_{j}\left(N+1, Q^{2}, \mu^{2}\right) \Delta_{i j \rightarrow k l, I}^{(\mathrm{int})}\left(N+1, Q^{2}, \mu^{2}\right) .
\end{aligned}
$$

The functions $\Delta_{i}\left(N+1, Q^{2}, \mu^{2}\right)$ resumming the collinear or collinear and soft radiation from the incoming partons are universal and their form can be found e.g. in [7]. The new elements which have to be calculated in order to obtain the resummed predictions from (2.2) are the LO partonic cross-sections in $N$-space, $\tilde{\sigma}_{i j \rightarrow k l, I}^{(0)}\left(N, \alpha_{\mathrm{s}}\left(\mu^{2}\right)\right)$, and the soft function $\Delta_{i j \rightarrow k l, I}^{(\mathrm{int})}\left(N+1, Q^{2}, \mu^{2}\right)$. Both types of contributions are dependent on the colour configuration of the underlying hard-scattering $i j \rightarrow k l$ and have to be calculated separately for each colour exchange channel, indicated by the index $I$. We perform the calculations in the $s$-channel orthogonal colour basis.

The soft function, describing large-angle soft gluon emission, at the NLL accuracy is given by

$$
\ln \Delta_{i j \rightarrow k l, I, N}^{\text {(int) }}=\int_{0}^{1} d z \frac{z^{N-1}-1}{1-z} \frac{\alpha_{s}\left(4 m^{2}(1-z)^{2}\right)}{\pi} D_{i j \rightarrow k l, I}
$$

The coefficients $D_{i j \rightarrow k l, I}$ are related to the threshold limit $\beta \rightarrow 0$ of the one-loop anomalous dimension matrices $\Gamma_{i j \rightarrow k l}$. In this limit, the matrices calculated in the $s$-channel orthogonal colour basis become diagonal. Each diagonal element $\Gamma_{i j \rightarrow k l, I I}$ contributes to the corresponding $D_{i j \rightarrow k l, I}$ coefficient. The expressions for $\Gamma_{i j \rightarrow k l}$ in the case $k l=\tilde{q} \tilde{q}$ can be found in [14], $k l=\tilde{g} \tilde{g}$ in [6, 7] and $k l=\tilde{q} \tilde{q}, \tilde{q} \tilde{g}$ in [8]. The corresponding expressions for the LO $N$-space colour contributions and the $D_{i j \rightarrow k l, I}$ coefficients have been calculated in [6,7] and in [8] for the $\tilde{q} \overline{\tilde{q}}, \tilde{g} \tilde{g}$ and $\tilde{q} \tilde{q}, \tilde{q} \tilde{g}$ production, respectively. We list the $D_{i j \rightarrow k l, I}$ coefficients for all the subprocesses $i j \rightarrow k l$ of interest in Table 1. The values of the coefficients equal the negative values of the quadratic Casimir operators belonging to the irreducible $\mathrm{SU}(3)$ representations for the outgoing state in a given process. This confirms the physical picture of the soft gluon radiation at production threshold being governed by the total colour charge of the heavy-particle pair in the final state $[10,16]$. 


$$
\begin{aligned}
& D_{q \bar{q} \rightarrow \tilde{q} \overline{\tilde{q}}, I}^{(1)}=\{0,-3\} \quad I=\{\mathbf{1}, \mathbf{8}\} \\
& D_{g g \rightarrow \tilde{q} \overline{\tilde{q}}, I}^{(1)}=\{0,-3\} \quad I=\{\mathbf{1}, \mathbf{8}\} \\
& D_{q \bar{q} \rightarrow \tilde{g} \tilde{g}, I}^{(1)}=\{0,-3,-3\} \quad I=\left\{\mathbf{1}, \mathbf{8}_{\mathbf{S}}, \mathbf{8}_{\mathbf{A}}\right\} \\
& D_{g g \rightarrow \tilde{g} \tilde{g}, I}^{(1)}=\{0,-3,-3,-6,-8\} \quad I=\left\{\mathbf{1}, \mathbf{8}_{\mathbf{S}}, \mathbf{8}_{\mathbf{A}}, \mathbf{1 0} \oplus \overline{\mathbf{1 0}}, 27\right\} \\
& D_{q q \rightarrow \tilde{q} \tilde{q}, I}^{(1)}=\{-4 / 3,-10 / 3\} \quad I=\{\overline{\mathbf{3}}, \mathbf{6}\} \\
& D_{q g \rightarrow \tilde{q} \tilde{g}, I}^{(1)}=\{-4 / 3,-10 / 3,-16 / 3\} \quad I=\{\mathbf{3}, \overline{\mathbf{6}}, \mathbf{1 5}\}
\end{aligned}
$$

Table 1: The soft coefficients $D_{i j \rightarrow k l, I}$ together with the corresponding irreducible SU(3) representations spanned by the vectors of the colour basis.

\section{Numerical results}

The resummation-improved cross sections, $\sigma_{\mathrm{NLL}+\mathrm{NLO}}$, are obtained through matching the NLL-resummed results with the complete NLO cross sections, in the manner described e.g. in Ref [7, 8]. The NLO cross sections are evaluated using the PROSP INO code [18], based on calculations $[4,5]$. We assume the left-handed and right-handed squarks in the final state to be mass-degenerate and sum over chiralities. No top squarks in the final state are considered. In our calculations we use the MSTW 2008 NLO pdfs [19] and two-loop running strong coupling constant $\alpha_{\mathrm{s}}$ in the $\overline{\mathrm{MS}}$ scheme with five active flavours.

We find that the NLL corrections to hadroproduction of squarks and gluinos are positive and lead to higher predictions for the total cross sections compared to the NLO results. In general, the most significant effects arise for processes characterised by high contributions from gluon initial states and the presence of gluinos in the final states. This is to be expected due to the high colour charge and, correspondingly, the large values of the Casimir invariants involved. The impact of the NLL resummation on the total cross section for the inclusive squark and gluino production, $p p^{(-)} \rightarrow$ $\tilde{q} \tilde{\tilde{q}}, \tilde{q} \tilde{q}, \tilde{q} \tilde{g}, \tilde{g} \tilde{g}+X$, is illustrated in Fig. 1 . The relative $\mathrm{K}$-factor $K_{\mathrm{NLL}}-1 \equiv \sigma_{\mathrm{NLL}+\mathrm{NLO}} / \sigma_{\mathrm{NLO}}-1$, shown for the Tevatron in Fig. 1a) and for the LHC in Fig. 1b), is calculated for various values of a fixed ratio $r$ of squark and gluino mass, $r=m_{\tilde{g}} / m_{\tilde{q}}$. For the average mass $m=600 \mathrm{GeV}$ the correction to the inclusive cross section at the Tevatron due to NLL resummation can be as high as $18 \%$. The inclusive corrections are smaller at the LHC for sparticle masses below $3 \mathrm{TeV}$ (see Fig. 1b). Given the sparticle mass ranges that we consider, this is consistent with the fact that the distance from threshold, i.e. the value of the variable $1-\rho=1-4 \mathrm{~m}^{2} / S$, is on average larger at the LHC than at the Tevatron.

In Figs. 2a) and 2b) we show for the Tevatron and LHC, respectively, the resummed NLL+NLO total cross section for inclusive squark and gluino production as a function of the average sparticle mass $m$. For illustration we show these results for the choice $m_{\tilde{q}}=m_{\tilde{g}}$. The error bands indicate the theoretical uncertainty of the NLL+NLO total cross section due to the scale variation in the range $m / 2 \leq \mu \leq 2 m$. Both at the Tevatron and at the LHC, soft-gluon resummation leads to a reduction by around $30 \%$ in this part of the theoretical uncertainty. The results presented in Fig. 2 are the most accurate theoretical predictions currently available for the above processes.

\section{Acknowledgments}

This work has been supported in part by the Helmholtz Alliance "Physics at the Terascale", the 


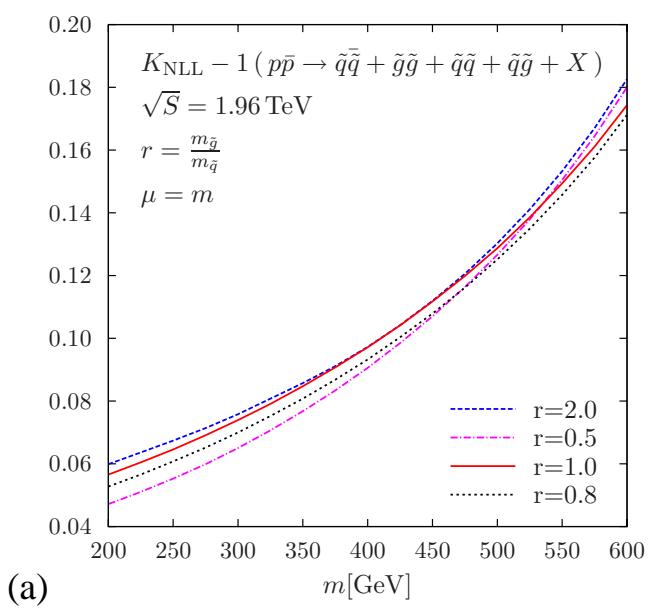

(b)

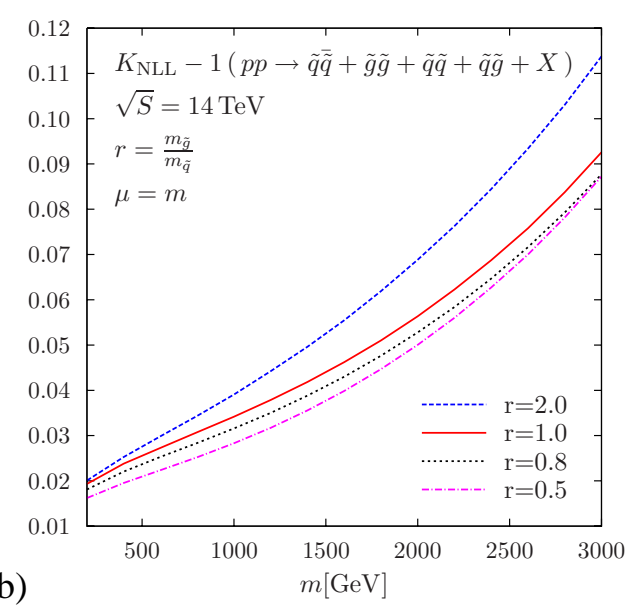

Figure 1: The relative NLL $K$-factor $K_{\mathrm{NLL}}-1=\sigma_{\mathrm{NLL}+\mathrm{NLO}} / \sigma_{\mathrm{NLO}}-1$ for the inclusive squark and gluino pair-production cross section, $p \bar{p} / p p \rightarrow \tilde{q} \tilde{q}+\tilde{q} \overline{\tilde{q}}+\tilde{q} \tilde{g}+\tilde{g} \tilde{g}+X$, at the Tevatron (a) and the LHC (b) as a function of the average sparticle mass $m$. Shown are results for various mass ratios $r=m_{\tilde{g}} / m_{\tilde{q}}$.

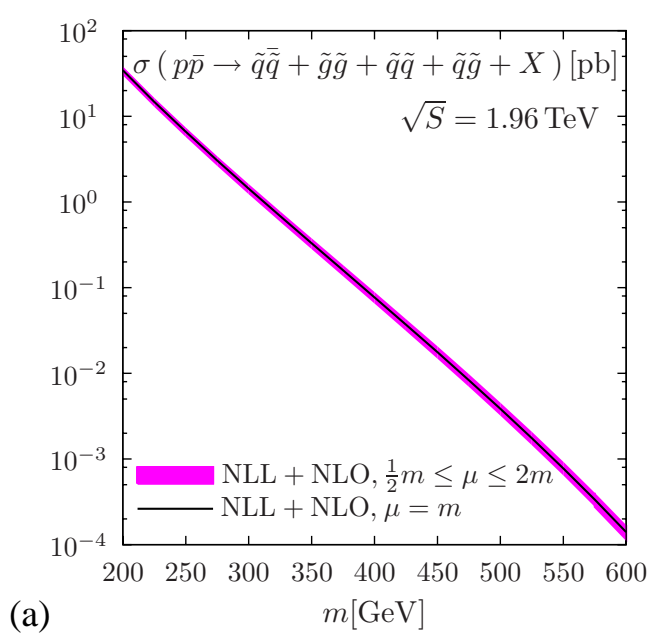

(b)

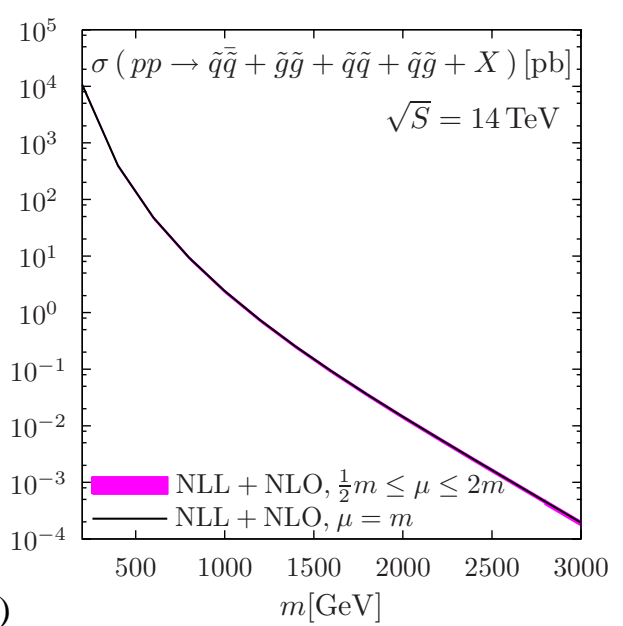

Figure 2: The NLL+NLO cross section for inclusive squark and gluino pair-production, $p \bar{p} / p p \rightarrow \tilde{q} \tilde{q}+$ $\tilde{q} \tilde{\tilde{q}}+\tilde{q} \tilde{g}+\tilde{g} \tilde{g}+X$, at the Tevatron (a) and the LHC (b) as a function of the average sparticle mass $m$. Shown are results for the mass ratio $r=m_{\tilde{g}} / m_{\tilde{q}}=1$. The error band corresponds to a variation of the common renormalization and factorization scale in the range $m / 2 \leq \mu \leq 2 m$.

DFG Graduiertenkolleg "Elementary Particle Physics at the TeV Scale", the Foundation for Fundamental Research of Matter (FOM), the National Organization for Scientific Research (NWO), the DFG SFB/TR9 “Computational Particle Physics", and the European Community's Marie-Curie Research Training Network under contract MRTN-CT-2006-035505 "Tools and Precision Calculations for Physics Discoveries at Colliders".

\section{References}

[1] T. Aaltonen et al. [CDF Collaboration], Phys. Rev. Lett. 102 (2009) 121801. 
[2] V. M. Abazov et al. [D0 Collaboration], Phys. Lett. B 660 (2008) 449.

[3] H. E. Haber and G. L. Kane, Phys. Rept. 117, 75 (1985).

[4] W. Beenakker, R. Höpker, M. Spira and P. M. Zerwas, Phys. Rev. Lett. 74, 2905 (1995); Z. Phys. C 69, 163 (1995).

[5] W. Beenakker, R. Höpker, M. Spira and P. M. Zerwas, Nucl. Phys. B 492, 51 (1997).

[6] A. Kulesza and L. Motyka, Phys. Rev. Lett. 102 (2009) 111802.

[7] A. Kulesza and L. Motyka, Phys. Rev. D 80 (2009) 095004.

[8] W. Beenakker, S. Brensing, M. Krämer, A. Kulesza, E. Laenen and I. Niessen, JHEP 12 (2009) 041.

[9] U. Langenfeld and S. O. Moch, Phys. Lett. B 675 (2009) 210.

[10] M. Beneke, P. Falgari and C. Schwinn, Nucl. Phys. B 828, (2010) 69; arXiv:0909.3488 [hep-ph].

[11] G. Sterman, Nucl. Phys. B 281 (1987) 310.

[12] S. Catani and L. Trentadue, Nucl. Phys. B 327 (1989) 323.

[13] H. Contopanagos, E. Laenen and G. Sterman, Nucl. Phys. B 484 (1997) 303.

[14] N. Kidonakis and G. Sterman, Nucl. Phys. B 505 (1997) 321.

[15] N. Kidonakis, G. Oderda and G. Sterman, Nucl. Phys. B 525 (1998) 299; Nucl. Phys. B 531 (1998) 365 .

[16] R. Bonciani, S. Catani, M. L. Mangano and P. Nason, Nucl. Phys. B 529, 424 (1998).

[17] S. Catani, M. L. Mangano, P. Nason and L. Trentadue, Nucl. Phys. B 478 (1996) 273.

[18] see http://www.thphys.uni-heidelberg.de/ plehn/prospino/ or http://people.web.psi.ch/spira/prospino/

[19] A. D. Martin, W. J. Stirling, R. S. Thorne and G. Watt, Eur. Phys. J. C 63 (2009) 189. 